\title{
Low grade B-cell leukemia/lymphoma with macrogloburinemia
}

Keywords: Splenic low grade B-cell lymphoma, Atypical chronic lymphoid leukemia, Macrogloburinemia

\section{TO THE EDITOR}

A 64-year-old male was referred to our hospital due to persistent leukocytosis for over two years. He did not have any B symptoms, hepatosplenomegaly, or lymphadenopathy. Peripheral blood (PB) demonstrated hemoglobin of $11.3 \mathrm{~g} / \mathrm{dL}$ (mean corpuscular volume 85.2 ), platelets of $291 \times 10^{9} / \mathrm{L}$, and leukocytes of $37.2 \times 10^{9} / \mathrm{L}$ with $13 \%$ neutrophils, $0.5 \%$ eosinophils, $0.5 \%$ basophils, $1.5 \%$ monocytes, and $84.5 \%$ lymphocytes. Approximately $90 \%$ of these lymphocytes were large atypical lymphocytes (Figure 1a). Serological test results were as follows: total protein $7.3 \mathrm{~g} / \mathrm{dL}$, albumin $2.7 \mathrm{~g} / \mathrm{dL}$, $\mathrm{IgG} / \mathrm{A} / \mathrm{M} 565 / 104 / 3447 \mathrm{mg} / \mathrm{dL}$ with M-peak on electrophoresis, lactate dehydrogenase $185 \mathrm{U} / \mathrm{L}$, and soluble interleukin-2 receptor $2973 \mathrm{U} / \mathrm{mL}$. Serum electrolytes, and renal and liver function tests were within normal limits.

Flow cytometry analysis of $\mathrm{PB}$ revealed that the population of lymphocytes with expression of CD5, 19, 20, 25, FMC-7, and strong immunoglobulin kappa chain restriction were negative for CD10 and CD23. Chromosomal analysis exhibited 47,X,-Y,+der(3)t(3;14)(q27;q32),t(3;14), $+12[7] / 46, X Y[8]$, which suggested correlation of IgH/BCL6. Although FISH analysis was 78\% positive for trisomy 12 , BCL6 split signal, and del(13q), del(17p) was negative.

Bone marrow aspiration showed hypercellular marrow with $80 \%$ atypical lymphoid cells, the same as observed in PB. Hematoxylin and eosin stained sections of the BM biopsy specimen demonstrated hypercellular BM with proliferation of small atypical lymphoid cells having irregular nuclei and abundant cytoplasm (Figure 1b, c).

Immunohistochemistry was positive for CD20, CD79a, bcl-2, CD5 (weak), MUM-1 (weak), whereas CD3, Cyclin D1, CD10, bcl-6, SOX-11, LEF-1, kappa, and lambda were negative, and Ki-67 was weakly positive (Figure 1d-f). Flow cytometry analysis of BM demonstrated the same phenotype as BM, and additionally showed positivity for CD22 and was weak for CD13 and CD11c. However, chromosomal analysis of $\mathrm{BM}$ was as follows and revealed a discrepancy with PB:45,X,-Y[3]/46,XY,del(20)(q1?)[2]/46,XY, $\mathrm{t}(11 ; 14)(\mathrm{q} 13 ; \mathrm{q} 32)[1] / 46, X Y[14]$. FISH analysis for the same BM specimen was negative for IgH-BCL1 fusion. Furthermore, MYD88 mutation was also negative.

Finally, as the phenotype of the tumor cells was atypical for any low grade lymphoma subtype, he was diagnosed with atypical CLL/ splenic low grade B-cell lymphoma. Systemic CT scanning showed only mild splenomegaly without nodal lesion, and because he had no symptoms or organ dysfunction, he received watchful waiting without significant progression over a year.

\section{CONFLICT OF INTEREST}

The authors declare no conflict of interest in this study.

Hiroyuki Takahashi, ${ }^{1)}$ Makiko Enaka ${ }^{2)}$

${ }^{1)}$ Department of Hematology and Clinical Immunology, Yokohama City University School of Medicine,

${ }^{2}$ Department of Pathology, Yokohama City University

Hospital

Corresponding author: Hiroyuki Takahashi Department of Hematology and Clinical Immunology, Yokohama City University School of Medicine 3-9, Fukumura, Kanazawa, Yokohama, Kanagawa, Japan E-mail: hirotak-ycu01@umin.ac.jp

Received: January 30, 2017.

Revised: April 27, 2017.

Accepted: May 12, 2017. 
Takahashi H, et al.

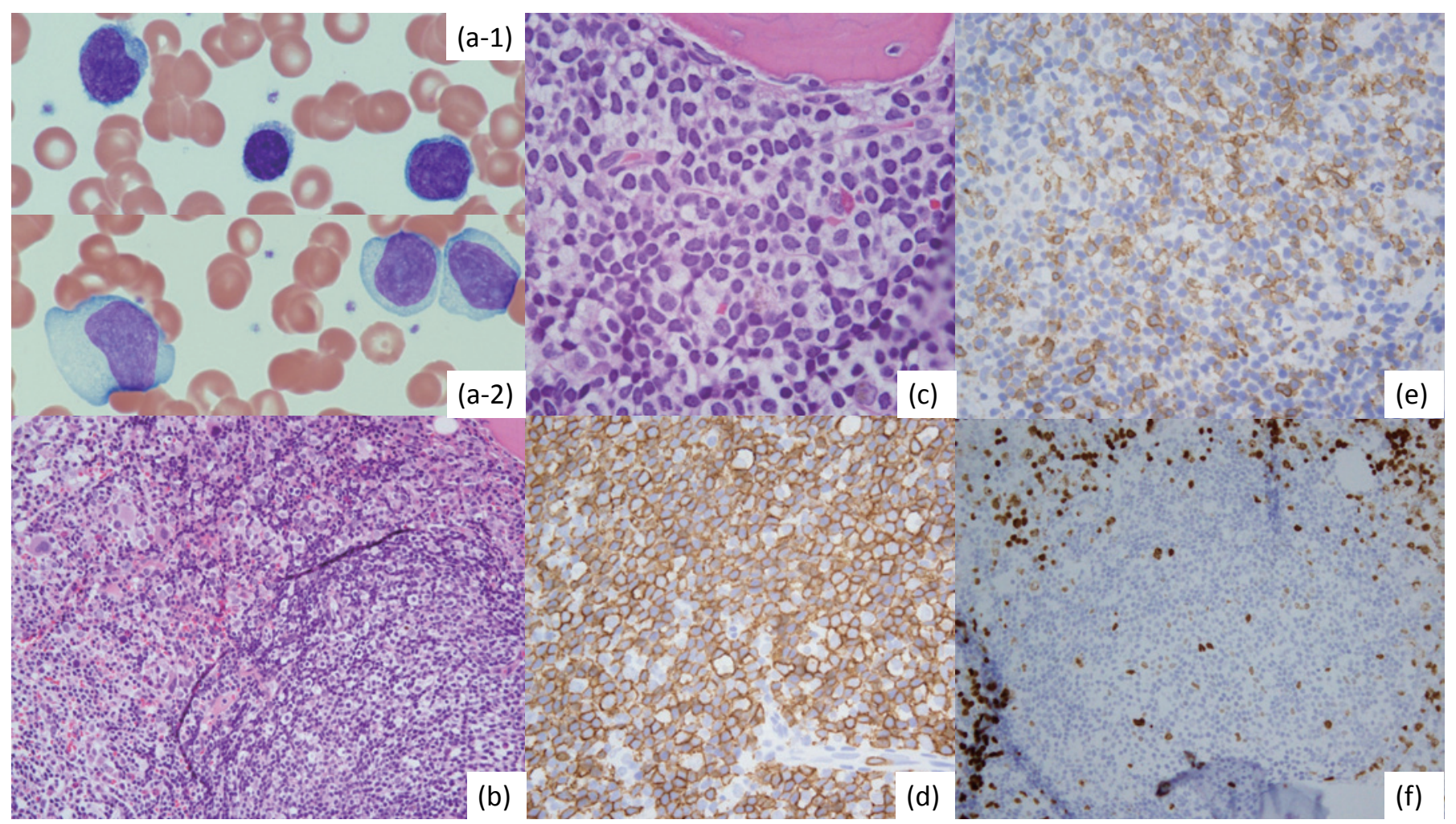

Fig. 1. Findings of peripheral blood smear specimen and formalin fixed paraffin embedded bone marrow biopsy specimen.

Wright-Giemsa stain of peripheral blood smear created by natural drying $(\boldsymbol{a}-\mathbf{1})$ and forced drying $(\boldsymbol{a}-2)$ showed proliferation of large atypical lymphocytes. Bone marrow exhibited hypercellular BM with proliferation of small atypical lymphoid cells having irregular nuclei and abundant cytoplasm on $(\boldsymbol{b})$ low magnification and $(\boldsymbol{c})$ high magnification views (hematoxylin and eosin staining). By immunohistochemical analyses, the atypical cells were $(\boldsymbol{d})$ positive for CD20, and $(\boldsymbol{e})$ weakly positive for CD5 and $(\boldsymbol{f}) \mathrm{Ki}-67$. 


\section{EXPERT'S COMMENT}

The diagnosis of low-grade B cell leukemia/lymphoma is often not easy because there are a few cases that exist in these boundary areas. ${ }^{1,2}$ However, diseases such as chronic lymphocytic leukemia (CLL), splenic marginal zone lymphoma (SMZL), mantle cell lymphoma (MCL), and lymphoplasmacytic lymphoma (LPL) are well-defined.

The current case report details a patient with low-grade B cell leukemia/lymphoma with IgM monoclonal protein. Morphologically, according to a naturally dried specimen, many cells appeared to be matured small lymphocytes (a-1). The cell surface appeared slightly lumpy. In contrast, according to force-dried specimens, many cells were large lymphocytes with widespread cytoplasm (a-2). Considering the morphology, it seemed that there was no problem in diagnosing this case as CLL. ${ }^{3}$ Cell surface markers were CD5positive, but CD23-negative. In addition, lymphocytes in the bone marrow were CD13-positive. Chromosome analysis suggested the involvement of $\mathrm{IgH} / \mathrm{BCL} 2$, and trisomy 12 , which is detected more frequently in CLL, was observed.

In fact, we noted the presence of CD13-positive, unclassifiable, low-grade B cell leukemia/lymphoma in a national joint study of CLL. In this study, CD13-positivity was found in 32 of $153(21 \%)$ cases. The median age was 65 years and 29 patients $(91 \%)$ were male. There were 14 CLL patients with typical morphology, and 18 with atypical morphology. CD5- and CD23- negativity were found in 7 and 8 patients, respectively. All cases exhibited high expression of CD22. FMC7 expression was observed in $26(81 \%)$. Fluorescent in situ hybridization (FISH) analysis showed trisomy 12 in 6 of $23(26 \%)$ cases. IGHV was mutated in all analyzed cases. Although some cases had IgM monoclonal protein, differentiation into plasma cells was not detected.
Thus, we suggest that CD13-positive CLL-like cases could be a novel subtype of low-grade B cell leukemia with unique features. ${ }^{4}$

When viewed with this perspective, the age, sex, cell surface markers, and clinical features in this case were consistent with those of CD13-positive low-grade B cell leukemia. Conversely, there exists a possibility of some CLL cases being difficult to distinguish from leukemic MZL or LPL; thus, further evaluation is warranted.

\section{REFERENCES}

1 Challagundla P, Medeiros LJ, Kanagal-Shamanna R, Miranda RN, Jorgensen JL: Differential expression of CD200 in B-cell neoplasms by flow cytometry can assist in diagnosis, subclassification, and bone marrow staging. Am J Clin Pathol 142:837844, 2014

2 Aoki S: Chronic lymphocytic leukemia. Rinsho Ketsueki 55:213-222, 2014 Review. Japanese

3 Hallek M, Cheson BD, Catovsky D, Caligaris-Cappio F, Dighiero G, et al.: Guidelines for the diagnosis and treatment of chronic lymphocytic leukemia: a report from the International Workshop on Chronic Lymphocytic Leukemia updating the National Cancer Institute-Working Group 1996 guidelines. Blood 111: 5446-5456, 2008

4 Uchiyama T, Aoki S, Takizawa J, Nakamura N, Utsunomiya A, et al:: CD13 positive chronic lymphocytic leukemia: A distinct subset of CLL. Rinsho Ketsueki 57:1502, 2016 abst

Sadao Aoki

Department of Pathophysiology, Faculty of Pharmaceutical Sciences, Niigata University of Pharmacy and Applied Life Science E-mail: saoki@nupals.ac.jp 